\title{
GLOBAL WELL-POSEDNESS FOR THE 3D MAGNETO-MICROPOLAR EQUATIONS WITH FRACTIONAL DISSIPATION
}

\author{
BAO QUAN YUAN AND PAN PAN ZHANG \\ (Received 20 August, 2021)
}

\begin{abstract}
This paper focus on the Cauchy problem of the $3 \mathrm{D}$ incompressible magneto-micropolar equations with fractional dissipation in the Sobolev space. Liu, Sun and Xin obtained the global solutions to the 3D magneto-micropolar equations with $\alpha=\beta=\gamma=\frac{5}{4}$. Deng and Shang established the global well-posedness of the 3D magneto-micropolar equations in the case of $\alpha \geq \frac{5}{4}$, $\alpha+\beta \geq \frac{5}{2}$ and $\gamma \geq 2-\alpha \geq \frac{3}{4}$. In this paper, we establish the global wellposedness of the 3D magneto-micropolar equations with $\alpha=\beta=\frac{5}{4}$ and $\gamma=\frac{1}{2}$, which improves the results of Liu-Sun-Xin and Deng-Shang by reducing the value of $\gamma$ to $\frac{1}{2}$.
\end{abstract}

\section{Introduction}

In this paper, we are concerned with the global well-posedness to the $3 \mathrm{D}$ magneto-micropolar equations with fractional dissipation

$$
\left\{\begin{array}{l}
\partial_{t} u+u \cdot \nabla u+(\mu+\chi) \Lambda^{2 \alpha} u=-\nabla \pi+b \cdot \nabla b+2 \chi \nabla \times w, \\
\partial_{t} w+u \cdot \nabla w+4 \chi w-\kappa \nabla \nabla \cdot w+\eta \Lambda^{2 \gamma} w=2 \chi \nabla \times u, \\
\partial_{t} b+u \cdot \nabla b+\nu \Lambda^{2 \beta} b=b \cdot \nabla u, \\
\nabla \cdot u=\nabla \cdot b=0 \\
u(x, 0)=u_{0}(x), w(x, 0)=w_{0}(x), b(x, 0)=b_{0}(x),
\end{array}\right.
$$

where $u=u(x, t), w=w(x, t), b=b(x, t)$ and $\pi=\pi(x, t)$ represent the velocity of the fluid, the microrotational velocity, the magnetic field and the hydrostatic pressure, respectively. The parameters $\mu, \chi$ and $\frac{1}{\nu}$ are the kinematic viscosity, vortex viscosity and magnetic Reynolds number, respectively. $\kappa$ and $\eta$ are angular viscosities. The parameters $\alpha, \beta, \gamma$ are nonnegative constants, and $\Lambda=(-\Delta)^{\frac{1}{2}}$ denotes the Zygmund operator defined via Fourier transform, namely

$$
\widehat{\Lambda^{s} f}(\xi)=|\xi|^{s} \widehat{f}(\xi), \quad \forall s \geq 0 .
$$

The magneto-micropolar system (1.1) is closely related to many classical fluid equations. When the magnetic field disappears, namely $b=0$, then the system (1.1) reduces to the $3 \mathrm{D}$ incompressible micropolar equations which has been investigated extensively with many interesting results. The existence of weak solutions was proved by Galdi and Rionero in [5]. Yamaguchi [26] obtained the existence of global

2010 Mathematics Subject Classification 35Q35, 76W05, 35B65.

Key words and phrases: Magneto-micropolar equations; global well-posedness; fractional dissipation.

Part of this work is supported by the National Natural Science Foundation of China (No.11471103). 
strong solutions in a bounded domain. Recently, the hyperdissipation micropolar equations have received significant attention in mathematical fluid dynamics (see for example $[\mathbf{3}, \mathbf{7}, \mathbf{1 7}, \mathbf{2 1}])$.

When $w=0$ and $\chi=0$, the system (1.1) becomes the generalized magnetohydrodynamics (MHD) equations which model the motion of electrically conducting fluid. The global well-posedness of the MHD equations have been investigated extensively and important progress has been made. Readers may refer to $[\mathbf{1 2}, \mathbf{1 3}, \mathbf{2 2}, \mathbf{2 3}, \mathbf{2 5}]$ and the references therein.

For the magneto-micropolar equations (1.1) in $\mathbb{R}^{2}$, Regmi and Wu [15] established the global regularity for magneto-micropolar equations with partial dissipation. Ma [11] extended the results of Regmi and $\mathrm{Wu}$ to other mixed partial viscosities cases. Yamazaki [27] established the global regularity of solutions for 2D magneto-micropolar equations with $\alpha=\beta=1, \gamma=0$. Recently, Shang and Zhao obtained the global regularity with $\alpha=0, \beta>1, \gamma=1$ in [19]. Shang and Wu [18] dealt with the global regularity problem with $1<\alpha<2,0<\beta<1, \alpha+\beta \geq 2$, $\gamma=0$ or $\alpha=2, \beta=\gamma=0$, or $\alpha>0, \beta=\gamma=1$. Many more exciting results on the global regularity for magneto-micropolar equations with partial dissipation are available for the $2 \mathrm{D}$ case (see for example $[\mathbf{9}, \mathbf{1 4}, \mathbf{1 6}, \mathbf{3 1}, \mathbf{3 2}]$ ).

For the 3D case of the system (1.1), Yuan [28] first established the regularity of weak solutions and blow-up criteria for smooth solutions in the whole space. Gala extended the results of Yuan in [28] to the Morrey-Campanato spaces in [4]. The regularity criterion for the 3D magneto-micropolar fluid equations in TriebelLizorkin space was proved in [34]. For more blow-up criteria of smooth solutions and the regularity criteria of weak solutions readers refer to $[\mathbf{2 4}, \mathbf{2 9}, \mathbf{3 0}, 33]$. Recently, Li-Shang [8] and Tan-Wu [20] established the existence of 3D small global smooth solutions in the case of $\alpha=\beta=\gamma=1$. Liu, Sun and Xin [10] obtained the global existence and uniqueness of solutions for the case $\alpha=\beta=\gamma=\frac{1}{2}+\frac{n}{4}$ with $n \geq 3$. Very recently, Deng and Shang [2] established the global well-posedness of magneto-micropolar equations with $\alpha \geq \frac{1}{2}+\frac{n}{4}, \alpha+\gamma \geq \max \left\{2, \frac{n}{2}\right\}, \alpha+\beta \geq 1+\frac{n}{2}$ when $n \geq 3$.

In this paper, we consider the system (1.1) in the case of $\alpha=\beta=\frac{5}{4}$ and $\gamma=\frac{1}{2}$. We will show the global well-posedness of the following system

$$
\left\{\begin{array}{l}
\partial_{t} u+u \cdot \nabla u+(\mu+\chi) \Lambda^{\frac{5}{2}} u=-\nabla \pi+b \cdot \nabla b+2 \chi \nabla \times w \\
\partial_{t} w+u \cdot \nabla w+4 \chi w-\kappa \nabla \nabla \cdot w+\eta \Lambda w=2 \chi \nabla \times u \\
\partial_{t} b+u \cdot \nabla b+\nu \Lambda^{\frac{5}{2}} b=b \cdot \nabla u \\
\nabla \cdot u=\nabla \cdot b=0 \\
u(x, 0)=u_{0}(x), w(x, 0)=w_{0}(x), b(x, 0)=b_{0}(x) .
\end{array}\right.
$$

More precisely, we establish the following main result.

Theorem 1.1. Assume the initial data $\left(u_{0}, w_{0}, b_{0}\right) \in H^{2}\left(\mathbb{R}^{3}\right)$ with $\nabla \cdot u_{0}=\nabla \cdot b_{0}=$ 0 . Then the system (1.2) has a unique global solution $(u, w, b)$ satisfying

$$
(u, w, b) \in L^{\infty}\left(0, T ; H^{2}\left(\mathbb{R}^{3}\right)\right),(u, b) \in L^{2}\left(0, T ; H^{\frac{13}{4}}\left(\mathbb{R}^{3}\right)\right), w \in L^{2}\left(0, T ; H^{\frac{5}{2}}\left(\mathbb{R}^{3}\right)\right) .
$$
for any $T>0$.

Remark 1.2. Theorem 1.1 improves the results in $[\mathbf{1 0}]$ and $[\mathbf{2}]$ by reducing the value of $\gamma$ to $\gamma=\frac{1}{2}$. In fact, when $n=3$, Liu etc. [10] obtained the well-posedness of 
solutions to the system (1.1) with $\alpha=\beta=\gamma=\frac{5}{4}$. Deng and Shang [2] established the global well-posedness for the case of $\alpha \geq \frac{5}{4}, \alpha+\beta \geq \frac{5}{2}$ and $\gamma \geq 2-\alpha \geq \frac{3}{4}$. In Theorem 1.1 we only need $\gamma=\frac{1}{2}$.

Remark 1.3. Notice that the value of $\alpha=\beta=\frac{5}{4}$ and $\gamma=\frac{1}{2}$ here are the minimum, therefore Theorem 1.1 is also valid for the case of $\alpha=\beta \geq \frac{5}{4}$ and $\gamma \geq \frac{1}{2}$.

Remark 1.4. When the magnetic field $b=0$, system (1.2) reduces to the micropolar equations, the result of Theorem 1.1 also holds true for the micropolar equations.

Throughout the paper, $C$ stands for a generic positive constant which may be different from line to line. In the following, for notational convenience, we use $\|\cdot\|_{X}$ to denote $\|\cdot\|_{X\left(\mathbb{R}^{3}\right)}$. Furthermore, we use $\|(u, w, b)(t)\|_{X}^{p}$ to denote $\|u(t)\|_{X}^{p}+$ $\|w(t)\|_{X}^{p}+\|b(t)\|_{X}^{p}$.

\section{Preliminaries}

In this section we present several elementary lemmas which are needed in the proof of Theorem 1.1. The first contains two calculus inequalities involving fractional differential operators. We can find the details in [6] for example.

Lemma 2.1. Suppose that $s>0$ and $p \in(1, \infty)$. Let $f, g$ be two functions such that $\Lambda^{s} f \in L^{p_{1}}, g \in L^{p_{2}}, \Lambda^{s-1} g \in L^{p_{3}}$ and $\nabla f \in L^{p_{4}}$, then there exists a constant $C$ such that

$$
\left\|\left[\Lambda^{s}, f\right] g\right\|_{L^{p}} \leq C\left(\left\|\Lambda^{s} f\right\|_{L^{p_{1}}}\|g\|_{L^{p_{2}}}+\left\|\Lambda^{s-1} g\right\|_{L^{p_{3}}}\|\nabla f\|_{L^{p_{4}}}\right)
$$

with $p_{2}, p_{4} \in[1, \infty], p_{1}, p_{3} \in(1, \infty)$ such that

$$
\frac{1}{p}=\frac{1}{p_{1}}+\frac{1}{p_{2}}=\frac{1}{p_{3}}+\frac{1}{p_{4}}
$$

where $\left[\Lambda^{s}, f\right] g:=\Lambda^{s}(f g)-f\left(\Lambda^{s} g\right)$ and $\Lambda^{s}=(-\Delta)^{\frac{s}{2}}$. In particular, we have the following form of commutator estimate

$$
\left\|\left[\Lambda^{s-1} \partial_{i}, f\right] g\right\|_{L^{p}} \leq C\left(\|\Lambda f\|_{L^{p_{1}}}\left\|\Lambda^{s-1} g\right\|_{L^{p_{2}}}+\left\|\Lambda^{s} f\right\|_{L^{p_{3}}}\|g\|_{L^{p_{4}}}\right) .
$$
2.44].

Then we recall the known Gagliardo-Nirenberg inequality as follows [1, Theorem

Lemma 2.2. (Gagliardo-Nirenberg inequality) Let $1<q, r \leq \infty$ and $0<m<n<$ $\infty$. Then a constant $C$ exists such that

$$
\|f\|_{\dot{W}_{p}^{m}} \leq C\|f\|_{L^{q}}^{\alpha}\|f\|_{\dot{W}_{r}^{n}}^{1-\alpha} \text { with } \frac{1}{p}=\frac{\alpha}{q}+\frac{1-\alpha}{r} \text { and } \alpha=1-\frac{m}{n} .
$$

\section{Proof of the Main Theorem}

This section is devoted to the proof of Theorem 1.1. We first prove the global existence part. To this end, the crucial piece is the global a priori $H^{2}$ bound for $(u, w, b)$. If we obtain this global bound, then the existence part of Theorem 1.1 can be proved by the standard Friedrichs method, so we omit the details here. Thus our main effort is to establish the global bounds for $\|(u, w, b)(t)\|_{H^{2}\left(\mathbb{R}^{3}\right)}$ with any given $t>0$. 
3.1. The $L^{2}$ estimate. Taking the $L^{2}$ inner products of the system $(1.2)_{1,2,3}$ with $(u, w, b)$ respectively, adding the results and integrating by parts, it follows by $\nabla \cdot u=0$ and $\nabla \cdot b=0$ that

$$
\begin{aligned}
& \quad \frac{1}{2} \frac{\mathrm{d}}{\mathrm{d} t}\|(u, w, b)(t)\|_{L^{2}}^{2}+(\mu+\chi)\left\|\Lambda^{\frac{5}{4}} u\right\|_{L^{2}}^{2}+4 \chi\|w\|_{L^{2}}^{2}+\kappa\|\nabla \cdot w\|_{L^{2}}^{2} \\
& \quad+\eta\left\|\Lambda^{\frac{1}{2}} w\right\|_{L^{2}}^{2}+\nu\left\|\Lambda^{\frac{5}{4}} b\right\|_{L^{2}}^{2} \\
& =4 \chi \int_{\mathbb{R}^{3}} \nabla \times u \cdot w \mathrm{~d} x,
\end{aligned}
$$

where we have used the facts

$$
\int_{\mathbb{R}^{3}} b \cdot \nabla b \cdot u \mathrm{~d} x+\int_{\mathbb{R}^{3}} b \cdot \nabla u \cdot b \mathrm{~d} x=0, \quad \int_{\mathbb{R}^{3}} \nabla \times w \cdot u \mathrm{~d} x=\int_{\mathbb{R}^{3}} \nabla \times u \cdot w \mathrm{~d} x .
$$

Then applying Hölder inequality, Lemma 2.2 and Young's inequality, we derive that

$$
\begin{aligned}
4 \chi \int_{\mathbb{R}^{3}} \nabla \times u \cdot w \mathrm{~d} x & \leq 4 \chi\|\nabla u\|_{L^{2}}\|w\|_{L^{2}} \\
& \leq 4 \chi\|u\|_{L^{2}}^{\frac{1}{5}}\left\|\Lambda^{\frac{5}{4}} u\right\|_{L^{2}}^{\frac{4}{5}}\|w\|_{L^{2}} \\
& \leq 2 \chi\|w\|_{L^{2}}^{2}+\frac{(\mu+\chi)}{2}\left\|\Lambda^{\frac{5}{4}} u\right\|_{L^{2}}^{2}+C\|u\|_{L^{2}}^{2} .
\end{aligned}
$$

Inserting (3.2) into (3.1), it thus follows from the Gronwall's inequality that

$$
\begin{aligned}
& \|(u, w, b)(t)\|_{L^{2}}^{2}+(\mu+\chi) \int_{0}^{t}\left\|\Lambda^{\frac{5}{4}} u(\tau)\right\|_{L^{2}}^{2} d \tau+4 \chi \int_{0}^{t}\|w(\tau)\|_{L^{2}}^{2} \mathrm{~d} \tau \\
& \quad+2 \kappa \int_{0}^{t}\|\nabla \cdot w(\tau)\|_{L^{2}}^{2} \mathrm{~d} \tau+2 \eta \int_{0}^{t}\left\|\Lambda^{\frac{1}{2}} w(\tau)\right\|_{L^{2}}^{2} \mathrm{~d} \tau+2 \nu \int_{0}^{t}\left\|\Lambda^{\frac{5}{4}} b(\tau)\right\|_{L^{2}}^{2} \mathrm{~d} \tau \\
& \leq C .
\end{aligned}
$$

3.2. The $H^{\frac{3}{4}}$ estimate. Applying $\Lambda^{\frac{3}{4}}$ to the system $(1.2)_{1,3}$, dotting the resultant by $\left(\Lambda^{\frac{3}{4}} u, \Lambda^{\frac{3}{4}} b\right)$ respectively, integrating over $\mathbb{R}^{3}$ and adding them up, by the divergence free conditions $\nabla \cdot u=0$ and $\nabla \cdot b=0$, we have

$$
\begin{aligned}
& \quad \frac{1}{2} \frac{\mathrm{d}}{\mathrm{d} t}\left\|\left(\Lambda^{\frac{3}{4}} u, \Lambda^{\frac{3}{4}} b\right)(t)\right\|_{L^{2}}^{2}+(\mu+\chi)\left\|\Lambda^{2} u\right\|_{L^{2}}^{2}+\nu\left\|\Lambda^{2} b\right\|_{L^{2}}^{2} \\
& =\int_{\mathbb{R}^{3}}\left[\Lambda^{\frac{3}{4}}, b \cdot \nabla\right] b \cdot \Lambda^{\frac{3}{4}} u \mathrm{~d} x+2 \chi \int_{\mathbb{R}^{3}} \Lambda^{\frac{3}{4}}(\nabla \times w) \cdot \Lambda^{\frac{3}{4}} u \mathrm{~d} x \\
& \quad-\int_{\mathbb{R}^{3}}\left[\Lambda^{\frac{3}{4}}, u \cdot \nabla\right] u \cdot \Lambda^{\frac{3}{4}} u \mathrm{~d} x+\int_{\mathbb{R}^{3}}\left[\Lambda^{\frac{3}{4}}, b \cdot \nabla\right] u \cdot \Lambda^{\frac{3}{4}} b \mathrm{~d} x \\
& \quad-\int_{\mathbb{R}^{3}}\left[\Lambda^{\frac{3}{4}}, u \cdot \nabla\right] b \cdot \Lambda^{\frac{3}{4}} b \mathrm{~d} x \\
& :=I_{1}+I_{2}+I_{3}+I_{4}+I_{5},
\end{aligned}
$$

where the following property has been applied

$$
\int_{\mathbb{R}^{3}} b \cdot \nabla \Lambda^{\frac{3}{4}} b \cdot \Lambda^{\frac{3}{4}} u \mathrm{~d} x+\int_{\mathbb{R}^{3}} b \cdot \nabla \Lambda^{\frac{3}{4}} u \cdot \Lambda^{\frac{3}{4}} b \mathrm{~d} x=0 .
$$


By Hölder inequality, together with Lemma 2.1, Sobolev embedding theorem and Young's inequality, we find

$$
\begin{aligned}
I_{1} & \leq C\left\|\left[\Lambda^{\frac{3}{4}}, b \cdot \nabla\right] b\right\|_{L^{2}}\left\|\Lambda^{\frac{3}{4}} u\right\|_{L^{2}} \\
& \leq C\left\|\Lambda^{\frac{3}{4}} b\right\|_{L^{12}}\|\nabla b\|_{L^{\frac{12}{5}}}\left\|\Lambda^{\frac{3}{4}} u\right\|_{L^{2}} \\
& \leq C\left\|\Lambda^{2} b\right\|_{L^{2}}\left\|\Lambda^{\frac{5}{4}} b\right\|_{L^{2}}\left\|\Lambda^{\frac{3}{4}} u\right\|_{L^{2}} \\
& \leq \frac{\nu}{6}\left\|\Lambda^{2} b\right\|_{L^{2}}^{2}+C\left\|\Lambda^{\frac{5}{4}} b\right\|_{L^{2}}^{2}\left\|\Lambda^{\frac{3}{4}} u\right\|_{L^{2}}^{2} .
\end{aligned}
$$

Arguing similarly to above inequality (3.5), it can be derived that

$$
\begin{gathered}
I_{3} \leq C\left\|\left[\Lambda^{\frac{3}{4}}, u \cdot \nabla\right] u\right\|_{L^{2}}\left\|\Lambda^{\frac{3}{4}} u\right\|_{L^{2}} \\
\leq \frac{\mu+\chi}{8}\left\|\Lambda^{2} u\right\|_{L^{2}}^{2}+C\left\|\Lambda^{\frac{5}{4}} u\right\|_{L^{2}}^{2}\left\|\Lambda^{\frac{3}{4}} u\right\|_{L^{2}}^{2}, \\
I_{4} \leq C\left\|\left[\Lambda^{\frac{3}{4}}, b \cdot \nabla\right] u\right\|_{L^{2}}\left\|\Lambda^{\frac{3}{4}} b\right\|_{L^{2}} \\
\leq \frac{\mu+\chi}{8}\left\|\Lambda^{2} u\right\|_{L^{2}}^{2}+\frac{\nu}{6}\left\|\Lambda^{2} b\right\|_{L^{2}}^{2}+C\left(\left\|\Lambda^{\frac{5}{4}} u\right\|_{L^{2}}^{2}+\left\|\Lambda^{\frac{5}{4}} b\right\|_{L^{2}}^{2}\right)\left\|\Lambda^{\frac{3}{4}} b\right\|_{L^{2}}^{2}, \\
I_{5} \leq C\left\|\left[\Lambda^{\frac{3}{4}}, u \cdot \nabla\right] b\right\|_{L^{2}}\left\|\Lambda^{\frac{3}{4}} b\right\|_{L^{2}} \\
\leq \frac{\mu+\chi}{8}\left\|\Lambda^{2} u\right\|_{L^{2}}^{2}+\frac{\nu}{6}\left\|\Lambda^{2} b\right\|_{L^{2}}^{2}+C\left(\left\|\Lambda^{\frac{5}{4}} u\right\|_{L^{2}}^{2}+\left\|\Lambda^{\frac{5}{4}} b\right\|_{L^{2}}^{2}\right)\left\|\Lambda^{\frac{3}{4}} b\right\|_{L^{2}}^{2}
\end{gathered}
$$

By Hölder and Young's inequalities, it follows that

$$
\begin{aligned}
I_{2} & \leq 2 \chi\left\|\Lambda^{2} u\right\|_{L^{2}}\left\|\Lambda^{\frac{1}{2}} w\right\|_{L^{2}} \\
& \leq \frac{\mu+\chi}{8}\left\|\Lambda^{2} u\right\|_{L^{2}}^{2}+C\left\|\Lambda^{\frac{1}{2}} w\right\|_{L^{2}}^{2} .
\end{aligned}
$$

Inserting the estimates (3.5)-(3.9) into (3.4), it thus leads to

$$
\begin{aligned}
& \frac{\mathrm{d}}{\mathrm{d} t}\left\|\left(\Lambda^{\frac{3}{4}} u, \Lambda^{\frac{3}{4}} b\right)(t)\right\|_{L^{2}}^{2}+(\mu+\chi)\left\|\Lambda^{2} u\right\|_{L^{2}}^{2}+\nu\left\|\Lambda^{2} b\right\|_{L^{2}}^{2} \\
& \leq C\left(\left\|\Lambda^{\frac{5}{4}} u\right\|_{L^{2}}^{2}+\left\|\Lambda^{\frac{5}{4}} b\right\|_{L^{2}}^{2}\right)\left\|\left(\Lambda^{\frac{3}{4}} u, \Lambda^{\frac{3}{4}} b\right)\right\|_{L^{2}}^{2}+C\left\|\Lambda^{\frac{1}{2}} w\right\|_{L^{2}}^{2} .
\end{aligned}
$$

Then Gronwall's inequality and (3.3) imply that

$$
\left\|\left(\Lambda^{\frac{3}{4}} u, \Lambda^{\frac{3}{4}} b\right)(t)\right\|_{L^{2}}^{2}+(\mu+\chi) \int_{0}^{t}\left\|\Lambda^{2} u(\tau)\right\|_{L^{2}}^{2} \mathrm{~d} \tau+\nu \int_{0}^{t}\left\|\Lambda^{2} b(\tau)\right\|_{L^{2}}^{2} \mathrm{~d} \tau \leq C .
$$

3.3. The $H^{1}$ estimate. Applying $\Lambda$ to both sides of system $(1.2)_{1,2,3}$ and taking the $L^{2}$ inner products with $(\Lambda u, \Lambda w, \Lambda b)$ respectively, adding them up together with 
$\nabla \cdot u=0$ and $\nabla \cdot b=0$, we deduce

$$
\begin{aligned}
& \frac{1}{2} \frac{\mathrm{d}}{\mathrm{d} t}\|(\Lambda u, \Lambda w, \Lambda b)(t)\|_{L^{2}}^{2}+(\mu+\chi)\left\|\Lambda^{\frac{9}{4}} u\right\|_{L^{2}}^{2}+4 \chi\|\Lambda w\|_{L^{2}}^{2}+\kappa\|\Lambda \nabla \cdot w\|_{L^{2}}^{2} \\
& \quad+\eta\left\|\Lambda^{\frac{3}{2}} w\right\|_{L^{2}}^{2}+\nu\left\|\Lambda^{\frac{9}{4}} b\right\|_{L^{2}}^{2} \\
& =\int_{\mathbb{R}^{3}}[\Lambda, b \cdot \nabla] b \cdot \Lambda u \mathrm{~d} x+2 \chi \int_{\mathbb{R}^{3}} \Lambda(\nabla \times w) \cdot \Lambda u \mathrm{~d} x-\int_{\mathbb{R}^{3}}[\Lambda, u \cdot \nabla] u \cdot \Lambda u \mathrm{~d} x \\
& \quad+2 \chi \int_{\mathbb{R}^{3}} \Lambda(\nabla \times u) \cdot \Lambda w \mathrm{~d} x-\int_{\mathbb{R}^{3}}[\Lambda, u \cdot \nabla] w \cdot \Lambda w \mathrm{~d} x \\
& \quad+\int_{\mathbb{R}^{3}}[\Lambda, b \cdot \nabla] u \cdot \Lambda b \mathrm{~d} x-\int_{\mathbb{R}^{3}}[\Lambda, u \cdot \nabla] b \cdot \Lambda b \mathrm{~d} x \\
& :=J_{1}+J_{2}+J_{3}+J_{4}+J_{5}+J_{6}+J_{7},
\end{aligned}
$$

where we also take advantage of the cancellation identity

$$
\int_{\mathbb{R}^{3}} b \cdot \nabla \Lambda b \cdot \Lambda u \mathrm{~d} x+\int_{\mathbb{R}^{3}} b \cdot \nabla \Lambda u \cdot \Lambda b \mathrm{~d} x=0 .
$$

Use an argument similar to that used in deriving the estimate (3.5) to obtain that

$$
\begin{aligned}
J_{1} & \leq C\|[\Lambda, b \cdot \nabla] b\|_{L^{2}}\|\Lambda u\|_{L^{2}} \\
& \leq C\|\Lambda b\|_{L^{12}}\|\nabla b\|_{L^{\frac{12}{5}}}\|\Lambda u\|_{L^{2}} \\
& \leq C\left\|\Lambda^{\frac{9}{4}} b\right\|_{L^{2}}\left\|\Lambda^{\frac{5}{4}} b\right\|_{L^{2}}\|\Lambda u\|_{L^{2}} \\
& \leq \frac{\nu}{6}\left\|\Lambda^{\frac{9}{4}} b\right\|_{L^{2}}^{2}+C\left\|\Lambda^{\frac{5}{4}} b\right\|_{L^{2}}^{2}\|\Lambda u\|_{L^{2}}^{2} .
\end{aligned}
$$

Similarly, we have

$$
\begin{gathered}
J_{3} \leq C\|[\Lambda, u \cdot \nabla] u\|_{L^{2}}\|\Lambda u\|_{L^{2}} \\
\leq \frac{\mu+\chi}{6}\left\|\Lambda^{\frac{9}{4}} u\right\|_{L^{2}}^{2}+C\left\|\Lambda^{\frac{5}{4}} u\right\|_{L^{2}}^{2}\|\Lambda u\|_{L^{2}}^{2}, \\
J_{6} \leq C\|[\Lambda, b \cdot \nabla] u\|_{L^{2}}\|\Lambda b\|_{L^{2}} \\
\leq \frac{\mu+\chi}{6}\left\|\Lambda^{\frac{9}{4}} u\right\|_{L^{2}}^{2}+\frac{\nu}{6}\left\|\Lambda^{\frac{9}{4}} b\right\|_{L^{2}}^{2}+C\left(\left\|\Lambda^{\frac{5}{4}} u\right\|_{L^{2}}^{2}+\left\|\Lambda^{\frac{5}{4}} b\right\|_{L^{2}}^{2}\right)\|\Lambda b\|_{L^{2}}^{2}
\end{gathered}
$$

and

$$
\begin{aligned}
J_{7} & \leq C\|[\Lambda, u \cdot \nabla] b\|_{L^{2}}\|\Lambda b\|_{L^{2}} \\
& \leq \frac{\mu+\chi}{6}\left\|\Lambda^{\frac{9}{4}} u\right\|_{L^{2}}^{2}+\frac{\nu}{6}\left\|\Lambda^{\frac{9}{4}} b\right\|_{L^{2}}^{2}+C\left(\left\|\Lambda^{\frac{5}{4}} u\right\|_{L^{2}}^{2}+\left\|\Lambda^{\frac{5}{4}} b\right\|_{L^{2}}^{2}\right)\|\Lambda b\|_{L^{2}}^{2} .
\end{aligned}
$$

In view of the Hölder and Young's inequalities, one has

$$
\begin{aligned}
J_{2}+J_{4} & =4 \chi \int_{\mathbb{R}^{3}} \Lambda(\nabla \times u) \cdot \Lambda w \mathrm{~d} x \\
& \leq 4 \chi\left\|\Lambda^{2} u\right\|_{L^{2}}\|\Lambda w\|_{L^{2}} \\
& \leq(\mu+\chi)\left\|\Lambda^{2} u\right\|_{L^{2}}^{2}+C\|\Lambda w\|_{L^{2}}^{2} .
\end{aligned}
$$


By Hölder inequality, together with Sobolev embedding theorem and Young's inequality, it can be shown that

$$
\begin{aligned}
J_{5} & \leq C\|[\Lambda, u \cdot \nabla] w\|_{L^{2}}\|\Lambda w\|_{L^{2}} \\
& \leq C\|\Lambda u\|_{L^{6}}\|\nabla w\|_{L^{3}}\|\Lambda w\|_{L^{2}} \\
& \leq C\left\|\Lambda^{2} u\right\|_{L^{2}}\left\|\Lambda^{\frac{3}{2}} w\right\|_{L^{2}}\|\Lambda w\|_{L^{2}} \\
& \leq \frac{\eta}{2}\left\|\Lambda^{\frac{3}{2}} w\right\|_{L^{2}}^{2}+C\left\|\Lambda^{2} u\right\|_{L^{2}}^{2}\|\Lambda w\|_{L^{2}}^{2} .
\end{aligned}
$$

Collecting the estimates (3.12)-(3.17) into (3.11), we obtain

$$
\begin{aligned}
& \frac{\mathrm{d}}{\mathrm{d} t}\|(\Lambda u, \Lambda w, \Lambda b)(t)\|_{L^{2}}^{2}+(\mu+\chi)\left\|\Lambda^{\frac{9}{4}} u\right\|_{L^{2}}^{2}+8 \chi\|\Lambda w\|_{L^{2}}^{2}+2 \kappa\|\Lambda \nabla \cdot w\|_{L^{2}}^{2} \\
& \quad+\eta\left\|\Lambda^{\frac{3}{2}} w\right\|_{L^{2}}^{2}+\nu\left\|\Lambda^{\frac{9}{4}} b\right\|_{L^{2}}^{2} \\
& \leq C\left(1+\left\|\Lambda^{\frac{5}{4}} u\right\|_{L^{2}}^{2}+\left\|\Lambda^{\frac{5}{4}} b\right\|_{L^{2}}^{2}+\left\|\Lambda^{2} u\right\|_{L^{2}}^{2}\right)\|(\Lambda u, \Lambda w, \Lambda b)\|_{L^{2}}^{2}+(\mu+\chi)\left\|\Lambda^{2} u\right\|_{L^{2}}^{2} .
\end{aligned}
$$

Then Gronwall's inequality, together with (3.3) and (3.10) imply that

$$
\begin{aligned}
& \|(\Lambda u, \Lambda w, \Lambda b)(t)\|_{L^{2}}^{2}+(\mu+\chi) \int_{0}^{t}\left\|\Lambda^{\frac{9}{4}} u(\tau)\right\|_{L^{2}}^{2} \mathrm{~d} \tau+8 \chi \int_{0}^{t}\|\Lambda w(\tau)\|_{L^{2}}^{2} \mathrm{~d} \tau \\
& \quad+2 \kappa \int_{0}^{t}\|\Lambda \nabla \cdot w(\tau)\|_{L^{2}}^{2} \mathrm{~d} \tau+\eta \int_{0}^{t}\left\|\Lambda^{\frac{3}{2}} w(\tau)\right\|_{L^{2}}^{2} \mathrm{~d} \tau+\nu \int_{0}^{t}\left\|\Lambda^{\frac{9}{4}} b(\tau)\right\|_{L^{2}}^{2} \mathrm{~d} \tau \\
& \leq C .
\end{aligned}
$$

3.4. The $H^{2}$ estimate. Applying $\Lambda^{2}$ to both sides of system $(1.2)_{1,2,3}$, taking the $L^{2}$ inner products of the resulting equations with $\left(\Lambda^{2} u, \Lambda^{2} w, \Lambda^{2} b\right)$ respectively, and using the divergence free property, we deduce by adding them up

$$
\begin{aligned}
\frac{1}{2} & \frac{\mathrm{d}}{\mathrm{d} t}\left\|\left(\Lambda^{2} u, \Lambda^{2} w, \Lambda^{2} b\right)(t)\right\|_{L^{2}}^{2}+(\mu+\chi)\left\|\Lambda^{\frac{13}{4}} u\right\|_{L^{2}}^{2}+4 \chi\left\|\Lambda^{2} w\right\|_{L^{2}}^{2}+\kappa\left\|\Lambda^{2} \nabla \cdot w\right\|_{L^{2}}^{2} \\
& +\eta\left\|\Lambda^{\frac{5}{2}} w\right\|_{L^{2}}^{2}+\nu\left\|\Lambda^{\frac{13}{4}} b\right\|_{L^{2}}^{2} \\
= & \int_{\mathbb{R}^{3}}\left[\Lambda^{2}, b \cdot \nabla\right] b \cdot \Lambda^{2} u \mathrm{~d} x+2 \chi \int_{\mathbb{R}^{3}} \Lambda^{2}(\nabla \times w) \cdot \Lambda^{2} u \mathrm{~d} x-\int_{\mathbb{R}^{3}}\left[\Lambda^{2}, u \cdot \nabla\right] u \cdot \Lambda^{2} u \mathrm{~d} x \\
& +2 \chi \int_{\mathbb{R}^{3}} \Lambda^{2}(\nabla \times u) \cdot \Lambda^{2} w \mathrm{~d} x-\int_{\mathbb{R}^{3}}\left[\Lambda^{2}, u \cdot \nabla\right] w \cdot \Lambda^{2} w \mathrm{~d} x \\
& +\int_{\mathbb{R}^{3}}\left[\Lambda^{2}, b \cdot \nabla\right] u \cdot \Lambda^{2} b \mathrm{~d} x-\int_{\mathbb{R}^{3}}\left[\Lambda^{2}, u \cdot \nabla\right] b \cdot \Lambda^{2} b \mathrm{~d} x \\
:= & K_{1}+K_{2}+K_{3}+K_{4}+K_{5}+K_{6}+K_{7} .
\end{aligned}
$$

Arguing like in deriving (3.5), it follows that

$$
\begin{aligned}
K_{1} & \leq C\left\|\left[\Lambda^{2}, b \cdot \nabla\right] b\right\|_{L^{2}}\left\|\Lambda^{2} u\right\|_{L^{2}} \\
& \leq C\left\|\Lambda^{2} b\right\|_{L^{12}}\|\nabla b\|_{L^{\frac{12}{5}}}\left\|\Lambda^{2} u\right\|_{L^{2}} \\
& \leq C\left\|\Lambda^{\frac{13}{4}} b\right\|_{L^{2}}\left\|\Lambda^{\frac{5}{4}} b\right\|_{L^{2}}\left\|\Lambda^{2} u\right\|_{L^{2}} \\
& \leq \frac{\nu}{6}\left\|\Lambda^{\frac{13}{4}} b\right\|_{L^{2}}^{2}+C\left\|\Lambda^{\frac{5}{4}} b\right\|_{L^{2}}^{2}\left\|\Lambda^{2} u\right\|_{L^{2}}^{2} .
\end{aligned}
$$


Similarly, we obtain

$$
\begin{aligned}
K_{3} & \leq C\left\|\left[\Lambda^{2}, u \cdot \nabla\right] u\right\|_{L^{2}}\left\|\Lambda^{2} u\right\|_{L^{2}} \\
& \leq \frac{\mu+\chi}{10}\left\|\Lambda^{\frac{13}{4}} u\right\|_{L^{2}}^{2}+C\left\|\Lambda^{\frac{5}{4}} u\right\|_{L^{2}}^{2}\left\|\Lambda^{2} u\right\|_{L^{2}}^{2},
\end{aligned}
$$

$$
\begin{aligned}
K_{6} & \leq C\left\|\left[\Lambda^{2}, b \cdot \nabla\right] u\right\|_{L^{2}}\left\|\Lambda^{2} b\right\|_{L^{2}} \\
& \leq \frac{\mu+\chi}{10}\left\|\Lambda^{\frac{13}{4}} u\right\|_{L^{2}}^{2}+\frac{\nu}{6}\left\|\Lambda^{\frac{13}{4}} b\right\|_{L^{2}}^{2}+C\left(\left\|\Lambda^{\frac{5}{4}} u\right\|_{L^{2}}^{2}+\left\|\Lambda^{\frac{5}{4}} b\right\|_{L^{2}}^{2}\right)\left\|\Lambda^{2} b\right\|_{L^{2}}^{2}
\end{aligned}
$$

and

$$
\begin{aligned}
K_{7} & \leq C\left\|\left[\Lambda^{2}, u \cdot \nabla\right] b\right\|_{L^{2}}\left\|\Lambda^{2} b\right\|_{L^{2}} \\
& \leq \frac{\mu+\chi}{10}\left\|\Lambda^{\frac{13}{4}} u\right\|_{L^{2}}^{2}+\frac{\nu}{6}\left\|\Lambda^{\frac{13}{4}} b\right\|_{L^{2}}^{2}+C\left(\left\|\Lambda^{\frac{5}{4}} u\right\|_{L^{2}}^{2}+\left\|\Lambda^{\frac{5}{4}} b\right\|_{L^{2}}^{2}\right)\left\|\Lambda^{2} b\right\|_{L^{2}}^{2} .
\end{aligned}
$$

Thanks to Hölder inequality, Lemma 2.2 and Young's inequality, we have

$$
\begin{aligned}
K_{2}+K_{4} & =4 \chi \int_{\mathbb{R}^{3}} \Lambda^{2}(\nabla \times u) \cdot \Lambda^{2} w \mathrm{~d} x \\
& \leq 4 \chi\left\|\Lambda^{3} u\right\|_{L^{2}}\left\|\Lambda^{2} w\right\|_{L^{2}} \\
& \leq 4 \chi\left\|\Lambda^{2} u\right\|_{L^{2}}^{\frac{1}{5}}\left\|\Lambda^{\frac{13}{4}} u\right\|_{L^{2}}^{\frac{4}{5}}\left\|\Lambda^{2} w\right\|_{L^{2}} \\
& \leq \frac{\mu+\chi}{10}\left\|\Lambda^{\frac{13}{4}} u\right\|_{L^{2}}^{2}+C\left\|\left(\Lambda^{2} u, \Lambda^{2} w\right)\right\|_{L^{2}}^{2} .
\end{aligned}
$$

By Hölder inequality and Lemma 2.1, together with Sobolev embedding theorem and Young's inequality, we arrive at

$$
\begin{aligned}
K_{5} & \leq \int_{\mathbb{R}^{3}}\left[\Lambda^{2} \partial_{i}, u\right] w \cdot \Lambda^{2} w \mathrm{~d} x \\
& \leq C\left\|\left[\Lambda^{2} \partial_{i}, u\right] w\right\|_{L^{2}}\left\|\Lambda^{2} w\right\|_{L^{2}} \\
& \leq C\|\nabla u\|_{L^{12}}\left\|\Lambda^{2} w\right\|_{L^{\frac{12}{5}}}\left\|\Lambda^{2} w\right\|_{L^{2}}+C\left\|\Lambda^{3} u\right\|_{L^{\frac{12}{5}}}\|w\|_{L^{12}}\left\|\Lambda^{2} w\right\|_{L^{2}} \\
& \leq C\left\|\Lambda^{\frac{9}{4}} u\right\|_{L^{2}}\left\|\Lambda^{2} w\right\|_{L^{2}}^{\frac{3}{2}}\left\|\Lambda^{\frac{5}{2}} w\right\|_{L^{2}}^{\frac{1}{2}}+C\left\|\Lambda^{\frac{13}{4}} u\right\|_{L^{2}}\|w\|_{L^{2}}^{\frac{1}{6}}\left\|\Lambda^{\frac{3}{2}} w\right\|_{L^{2}}^{\frac{5}{6}}\left\|\Lambda^{2} w\right\|_{L^{2}} \\
& \leq \frac{\eta}{2}\left\|\Lambda^{\frac{5}{2}} w\right\|_{L^{2}}^{2}+\frac{\mu+\chi}{10}\left\|\Lambda^{\frac{13}{4}} u\right\|_{L^{2}}^{2}+C\left(1+\|w\|_{L^{2}}^{2}+\left\|\Lambda^{\frac{9}{4}} u\right\|_{L^{2}}^{2}+\left\|\Lambda^{\frac{3}{2}} w\right\|_{L^{2}}^{2}\right)\left\|\Lambda^{2} w\right\|_{L^{2}}^{2} .
\end{aligned}
$$

Substituting the estimates (3.20)-(3.25) into (3.19), we eventually obtain

$$
\begin{aligned}
& \frac{\mathrm{d}}{\mathrm{d} t}\left\|\left(\Lambda^{2} u, \Lambda^{2} w, \Lambda^{2} b\right)(t)\right\|_{L^{2}}^{2}+(\mu+\chi)\left\|\Lambda^{\frac{13}{4}} u\right\|_{L^{2}}^{2}+8 \chi\left\|\Lambda^{2} w\right\|_{L^{2}}^{2}+2 \kappa\left\|\Lambda^{2} \nabla \cdot w\right\|_{L^{2}}^{2} \\
& \quad+\eta\left\|\Lambda^{\frac{5}{2}} w\right\|_{L^{2}}^{2}+\nu\left\|\Lambda^{\frac{13}{4}} b\right\|_{L^{2}}^{2} \\
& \leq C\left(1+\|w\|_{L^{2}}^{2}+\left\|\Lambda^{\frac{5}{4}} u\right\|_{L^{2}}^{2}+\left\|\Lambda^{\frac{3}{2}} w\right\|_{L^{2}}^{2}+\left\|\Lambda^{\frac{5}{4}} b\right\|_{L^{2}}^{2}+\left\|\Lambda^{\frac{9}{4}} u\right\|_{L^{2}}^{2}\right)\left\|\left(\Lambda^{2} u, \Lambda^{2} w, \Lambda^{2} b\right)\right\|_{L^{2}}^{2} .
\end{aligned}
$$

Then Gronwall's inequality, together with (3.3) and (3.18) yield

$$
\begin{aligned}
& \left\|\left(\Lambda^{2} u, \Lambda^{2} w, \Lambda^{2} b\right)(t)\right\|_{L^{2}}^{2}+(\mu+\chi) \int_{0}^{t}\left\|\Lambda^{\frac{13}{4}} u(\tau)\right\|_{L^{2}}^{2} \mathrm{~d} \tau+8 \chi \int_{0}^{t}\left\|\Lambda^{2} w(\tau)\right\|_{L^{2}}^{2} \mathrm{~d} \tau \\
& \quad+2 \kappa \int_{0}^{t}\left\|\Lambda^{2} \nabla \cdot w(\tau)\right\|_{L^{2}}^{2} \mathrm{~d} \tau+\eta \int_{0}^{t}\left\|\Lambda^{\frac{5}{2}} w(\tau)\right\|_{L^{2}}^{2} \mathrm{~d} \tau+\nu \int_{0}^{t}\left\|\Lambda^{\frac{13}{4}} b(\tau)\right\|_{L^{2}}^{2} \mathrm{~d} \tau \\
& \leq C .
\end{aligned}
$$


Finally, we prove the uniqueness. Assume that $\left(u_{1}, w_{1}, b_{1}, \pi_{1}\right)$ and $\left(u_{2}, w_{2}, b_{2}, \pi_{2}\right)$ are two solutions for the system (1.2) with the same initial data. We define

$$
\bar{u} \triangleq u_{1}-u_{2}, \bar{w} \triangleq w_{1}-w_{2}, \bar{b} \triangleq b_{1}-b_{2}, \bar{\pi} \triangleq \pi_{1}-\pi_{2},
$$

thus the differences $(\bar{u}, \bar{w}, \bar{b}, \bar{\pi})$ between these two solutions satisfy the following equations

$$
\left\{\begin{array}{l}
\partial_{t} \bar{u}+u_{1} \cdot \nabla \bar{u}+\bar{u} \cdot \nabla u_{2}+(\mu+\chi) \Lambda^{\frac{5}{2}} \bar{u}=-\nabla \bar{\pi}+b_{1} \cdot \nabla \bar{b}+\bar{b} \cdot \nabla b_{2}+2 \chi \nabla \times \bar{w}, \\
\partial_{t} \bar{w}+u_{1} \cdot \nabla \bar{w}+\bar{u} \cdot \nabla w_{2}+4 \chi \bar{w}-\kappa \nabla \nabla \cdot \bar{w}+\eta \Lambda \bar{w}=2 \chi \nabla \times \bar{u}, \\
\partial_{t} \bar{b}+u_{1} \cdot \nabla \bar{b}+\bar{u} \cdot \nabla b_{2}+\nu \Lambda^{\frac{5}{2}} \bar{b}=b_{1} \cdot \nabla \bar{u}+\bar{b} \cdot \nabla u_{2}, \\
\nabla \cdot \bar{u}=\nabla \cdot \bar{w}=\nabla \cdot \bar{b}=0, \\
\bar{u}(x, 0)=\bar{w}(x, 0)=\bar{b}(x, 0)=0 .
\end{array}\right.
$$

Taking the $L^{2}$ inner products of the system $(3.27)_{1,2,3}$ with $(\bar{u}, \bar{w}, \bar{b})$ respectively, integrating by parts we obtain

$$
\begin{aligned}
& \frac{1}{2} \frac{\mathrm{d}}{\mathrm{d} t}\|(\bar{u}, \bar{w}, \bar{b})(t)\|_{L^{2}}^{2}+(\mu+\chi)\left\|\Lambda^{\frac{5}{4}} \bar{u}\right\|_{L^{2}}^{2}+4 \chi\|\bar{w}\|_{L^{2}}^{2}+\kappa\|\nabla \cdot \bar{w}\|_{L^{2}}^{2}+\eta\left\|\Lambda^{\frac{1}{2}} \bar{w}\right\|_{L^{2}}^{2} \\
& \quad+\nu\left\|\Lambda^{\frac{5}{4}} \bar{b}\right\|_{L^{2}}^{2} \\
& =\int_{\mathbb{R}^{3}} \bar{b} \cdot \nabla b_{2} \cdot \bar{u} \mathrm{~d} x+2 \chi \int_{\mathbb{R}^{3}} \nabla \times \bar{w} \cdot \bar{u} \mathrm{~d} x-\int_{\mathbb{R}^{3}} \bar{u} \cdot \nabla u_{2} \cdot \bar{u} \mathrm{~d} x+2 \chi \int_{\mathbb{R}^{3}} \nabla \times \bar{u} \cdot \bar{w} \mathrm{~d} x \\
& \quad-\int_{\mathbb{R}^{3}} \bar{u} \cdot \nabla w_{2} \cdot \bar{w} \mathrm{~d} x+\int_{\mathbb{R}^{3}} \bar{b} \cdot \nabla u_{2} \cdot \bar{b} \mathrm{~d} x-\int_{\mathbb{R}^{3}} \bar{u} \cdot \nabla b_{2} \cdot \bar{b} \mathrm{~d} x \\
& :=N_{1}+N_{2}+N_{3}+N_{4}+N_{5}+N_{6}+N_{7} .
\end{aligned}
$$

Applying Hölder inequality, Sobolev embedding theorem and Young's inequality, it then follows that

$$
\begin{aligned}
N_{1}+N_{7} & \leq 2\|\bar{b}\|_{L^{2}}\left\|\nabla b_{2}\right\|_{L^{\frac{12}{5}}}\|\bar{u}\|_{L^{12}} \\
& \leq 2\|\bar{b}\|_{L^{2}}\left\|\Lambda^{\frac{5}{4}} b_{2}\right\|_{L^{2}}\left\|\Lambda^{\frac{5}{4}} \bar{u}\right\|_{L^{2}} \\
& \leq \frac{(\mu+\chi)}{8}\left\|\Lambda^{\frac{5}{4}} \bar{u}\right\|_{L^{2}}^{2}+C\left\|\Lambda^{\frac{5}{4}} b_{2}\right\|_{L^{2}}^{2}\|\bar{b}\|_{L^{2}}^{2} .
\end{aligned}
$$

Similarly, it can be shown that

$$
\begin{aligned}
& N_{3} \leq C\|\bar{u}\|_{L^{12}}\left\|\nabla u_{2}\right\|_{L^{\frac{12}{5}}}\|\bar{u}\|_{L^{2}} \\
& \leq \frac{(\mu+\chi)}{8}\left\|\Lambda^{\frac{5}{4}} \bar{u}\right\|_{L^{2}}^{2}+C\left\|\Lambda^{\frac{5}{4}} u_{2}\right\|_{L^{2}}^{2}\|\bar{u}\|_{L^{2}}^{2}, \\
& N_{6} \leq C\|\bar{b}\|_{L^{12}}\left\|\nabla u_{2}\right\|_{L^{\frac{12}{5}}}\|\bar{b}\|_{L^{2}} \\
& \quad \leq \frac{\nu}{2}\left\|\Lambda^{\frac{5}{4}} \bar{b}\right\|_{L^{2}}^{2}+C\left\|\Lambda^{\frac{5}{4}} u_{2}\right\|_{L^{2}}^{2}\|\bar{b}\|_{L^{2}}^{2} .
\end{aligned}
$$


Again applying Hölder inequality, Lemma 2.2 and Young's inequality, it allows us to obtain

$$
\begin{aligned}
N_{2}+N_{4} & \leq 4 \chi\|\nabla \times \bar{u}\|_{L^{2}}\|\bar{w}\|_{L^{2}} \\
& \leq 4 \chi\|\bar{u}\|_{L^{2}}^{\frac{1}{5}}\left\|\Lambda^{\frac{5}{4}} \bar{u}\right\|_{L^{2}}^{\frac{4}{5}}\|\bar{w}\|_{L^{2}} \\
& \leq \frac{(\mu+\chi)}{8}\left\|\Lambda^{\frac{5}{4}} \bar{u}\right\|_{L^{2}}^{2}+C\|(\bar{u}, \bar{w})\|_{L^{2}}^{2}
\end{aligned}
$$

and

$$
\begin{aligned}
N_{5} & \leq C\|\bar{u}\|_{L^{6}}\left\|\nabla w_{2}\right\|_{L^{3}}\|\bar{w}\|_{L^{2}} \\
& \leq C\|\bar{u}\|_{L^{2}}^{\frac{1}{5}}\left\|\Lambda^{\frac{5}{4}} \bar{u}\right\|_{L^{2}}^{\frac{4}{5}}\left\|\Lambda^{\frac{3}{2}} w_{2}\right\|_{L^{2}}\|\bar{w}\|_{L^{2}} \\
& \leq \frac{(\mu+\chi)}{8}\left\|\Lambda^{\frac{5}{4}} \bar{u}\right\|_{L^{2}}^{2}+C\left(1+\left\|\Lambda^{\frac{3}{2}} w_{2}\right\|_{L^{2}}^{2}\right)\|(\bar{u}, \bar{w})\|_{L^{2}}^{2} .
\end{aligned}
$$

Putting the estimates (3.29)-(3.33) into (3.28), we finally arrive at

$$
\begin{aligned}
& \frac{\mathrm{d}}{\mathrm{d} t}\|(\bar{u}, \bar{w}, \bar{b})\|_{L^{2}}^{2}+(\mu+\chi)\left\|\Lambda^{\frac{5}{4}} \bar{u}\right\|_{L^{2}}^{2}+8 \chi\|\bar{w}\|_{L^{2}}^{2}+2 \kappa\|\nabla \cdot \bar{w}\|_{L^{2}}^{2} \\
& \quad+2 \eta\left\|\Lambda^{\frac{1}{2}} \bar{b}\right\|_{L^{2}}^{2}+\nu\left\|\Lambda^{\frac{5}{4}} \bar{b}\right\|_{L^{2}}^{2} \\
& \quad \leq C\left(1+\left\|\Lambda^{\frac{5}{4}} u_{2}\right\|_{L^{2}}^{2}+\left\|\Lambda^{\frac{3}{2}} w_{2}\right\|_{L^{2}}^{2}+\left\|\Lambda^{\frac{5}{4}} b_{2}\right\|_{L^{2}}^{2}\right)\|(\bar{u}, \bar{w}, \bar{b})\|_{L^{2}}^{2} .
\end{aligned}
$$

By the Gronwall's inequality, (3.3) and (3.18), we get the uniqueness immediately. Thus the proof of Theorem 1.1 is completed.

\section{References}

[1] H. Bahouri, J.-Y. Chemin and R. Danchin, Fourier Analysis and Nonlinear Partial Differential Equations, Grundlehren der Mathematischen Wissenschaften 343, Springer, Berlin, 2011.

[2] L. H. Deng and H. F. Shang, Global well-posedness for n-dimensional magnetomicropolar equations with hyperdissipation, Appl. Math. Lett. 111 (2021), Article ID 106610.

[3] B. Q. Dong and Z. F. Zhang, Global regularity of the 2D micropolar fluid flows with zero angular viscosity, J. Differ. Equations 249 (1) (2010), 200-213.

[4] S. Gala, Regularity criteria for the 3D magneto-micropolar fluid equations in the Morrey-Campanato space, NoDEA Nonlinear Differential Equations Appl. 17 (2) (2010), 181-194.

[5] G. P. Galdi and S. Rionero, A note on the existence and uniqueness of solutions of the micropolar fluid equations, Int. J. Engin. Sci. 15 (1977), 105-108.

[6] T. Kato and G. Ponce, Commutator estimates and the Euler and Navier-Stokes equations, Commun. Pure Appl. Math. 41 (7) (1988), 891-907.

[7] H. M. Li, Global regularity for the $3 D$ micropolar equations, Appl. Math. Lett. 92 (2019), 70-75.

[8] M. Li and H. F. Shang, Large time decay of solutions for the 3D magnetomicropolar equations, Nonlinear Anal. Real World Appl. 44 (2018), 479-496.

[9] H. X. Lin and Z. Y. Xiang, Global well-posedness for the 2D incompressible magneto-micropolar fluid system with partial viscosity, Sci. China Math. 63 (7) (2020), 1285-1306. 
[10] H. Liu, C. F. Sun and J. Xin, Well-posedness for the hyperviscous magnetomicropolar equations, Appl. Math. Lett. 107 (2020), Article ID 106403.

[11] L. L. Ma, On two-dimensional incompressible magneto-micropolar system with mixed partial viscosity, onlinear Anal. Real World Appl. 40 (2018), 95-129.

[12] W. G. Melo, T. Santos and P. R. Zingano, Global well-posedness of the $3 D$ generalized MHD equations in Lei-Lin-Gevrey and Lei-Lin spaces, Z. Angew. Math. Phys. 71 (6) (2020), Paper No. 195.

[13] C. X. Miao, B. Q. Yuan and B. Zhang, Well-posedness for the incompressible magneto-hydrodynamic system, Math. Methods Appl. Sci. 30 (8) (2007), 961976.

[14] D. Regmi, The 2D magneto-micropolar equations with partial dissipation, Math. Methods Appl. Sci. 42 (12) (2019), 4305-4317.

[15] D. Regmi and J. H. Wu, Global regularity for the $2 D$ magneto-micropolar equations with partial dissipation, J. Math. Study 49 (2) (2016), 169-194.

[16] H. F. Shang and C. W. Gu, Global regularity and decay estimates for $2 D$ magneto-micropolar equations with partial dissipation, Z. Angew. Math. Phys. 70 (3) (2019), Paper No. 85.

[17] H. F. Shang and M. Li, Global regularity for d-dimensional micropolar equations with fractional dissipation, Appl. Anal. 98 (8) (2019), 1576-1580.

[18] H. F. Shang and J. H. Wu, Global regularity for $2 D$ fractional magnetomicropolar equations, Math. Z. 297 (2021) (1-2), 775-802.

[19] H. F. Shang and J. F. Zhao, Global regularity for $2 D$ magneto-micropolar equations with only micro-rotational velocity dissipation and magnetic diffusion, Nonlinear Anal., Theory Methods Appl., Ser. A 150 (2017), 194-209.

[20] Z. Tan, W. P. Wu and J. F. Zhou, Global existence and decay estimate of solutions to magneto-micropolar fluid equations, J. Differ. Equations 266 (7) (2019), 4137-4169.

[21] D. H. Wang, J. H. Wu and Z. Ye, Global regularity of the three-dimensional fractional micropolar equations, J. Math. Fluid Mech. 22 (2) (2020), Paper No. 28.

[22] D. Y. Wei and Z. F. Zhang, Global well-posedness of the MHD equations via the comparison principle, Sci. China Math. 61 (11) (2018), 2111-2120.

[23] D. Y. Wei and Z. F. Zhang, Global Well-Posedness for the 2-D MHD Equations with Magnetic Diffusion, Commun. Math. Res. 36 (4) (2020), 377-389.

[24] F. Wu, A refined regularity criteria of weak solutions to the magneto-micropolar fluid equations, J. Evol. Equ. 21 (1) (2021), 725-734.

[25] J. H. Wu, Generalized MHD equations, J. Differ. Equations 195 (2) (2003), 284-312.

[26] N. Yamaguchi, Existence of global strong solution to the micropolar fluid equations, Math. Methods Appl. Sci. 28 (13) (2005), 1507-1526.

[27] K. Yamazaki, Global regularity of the two-dimensional magneto-micropolar fluid system with zero angular viscosity, Discrete Contin. Dyn. Syst. 35 (5) (2015), 2193-2207.

[28] B. Q. Yuan, Regularity of weak solutions to magneto-micropolar fluid equations, Acta Math. Sci. Ser. B Engl. Ed. 30 (5) (2010), 1469-1480. 
[29] B. Q. Yuan and X. Li, Regularity of weak solutions to the 3D magnetomicropolar equations in Besov spaces, Acta Appl. Math. 163 (1) (2019), 207223.

[30] B. Q. Yuan and X. Li, Blow-up criteria of smooth solutions to the threedimensional micropolar fluid equations in Besov space, Discrete Contin. Dyn. Syst., Ser. S 9 (6) (2016), 2167-2179.

[31] B. Q. Yuan and Y. Y. Qiao, Global regularity for the $2 D$ magneto-micropolar equations with partial and fractional dissipation, Comput. Math. Appl. 76 (2018), 2345-2359.

[32] B. Q. Yuan and Y. Y. Qiao, Global regularity of 2D Leray-alpha regularized incompressible magneto-micropolar equations, J. Math. Anal. Appl. 474 (1) (2019), 492-512.

[33] P. P. Zhang and B. Q. Yuan, An improved regularity criterion for the $3 D$ magneto-micropolar equations in homogeneous Besov space, J. Math. Anal. Appl. 499 (1) (2021), Article ID 125022.

[34] Z. J. Zhang, Z. A. Yao and X. F. Wang, A regularity criterion for the $3 D$ magneto-micropolar fluid equations in Triebel-Lizorkin spaces, Nonlinear Anal., Theory Methods Appl., Ser. A 74 (6) (2011), 2220-2225.

Bao Quan Yuan

Henan Polytechnic University,

Jiaozuo 454000,

Henan,

China

bqyuan@hpu.edu.cn
Pan Pan Zhang

Henan Polytechnic University,

Jiaozuo 454000,

Henan,

China

zhangpanpanmyl@163.com 